\title{
Towards the Development of a Modified Token based Congestion Control with Adaptive Forwarding for Opportunistic Networks
}

\author{
E. A. Adedokun \\ Faculty of Engineering \\ Department of Electrical and \\ Computer Engineering, \\ Ahmadu Bello University, Zaria, \\ Kaduna State-Nigeria
}

\author{
Shaibu Idris Salawu \\ Faculty of Engineering \\ Department of Electrical and \\ Computer Engineering, \\ Ahmadu Bello University, Zaria \\ Kaduna State-Nigeria
}

\author{
Basira Yahaya \\ Faculty of Engineering \\ Department of Electrical and \\ Computer Engineering, \\ Ahmadu Bello University, Zaria \\ Kaduna State-Nigeria
}

\begin{abstract}
In opportunistic networks the accessible network resources such a storage capacity, bandwidth, etc., are limited, and simply be consumed over time. This always result to network congestion, which has substantial impact on the overall network throughput. Due to the distinctive features of the network and the implementation of custody transfer mechanism, the conventional TCP congestion control mechanism fails in OppNets. The congestion control in OppNet has attracted the attention of the researchers because it attempts to establish network in an extreme environment where infrastructure is not obtainable, and when it succeeds, the message should not be dropped instantly since it has serious impact on the objective of OppNet. Instead, the message should be rerouted to the next best neighbor whose buffer is not full because some of the application areas are extremely sensitive. Some congestion control strategies have been considered compared and modified token-based congestion control algorithm is proposed by examining them.
\end{abstract}

\section{General Terms}

Opportunistic networks, Congestion control strategies and routing protocols

\section{Keywords}

Epidemic routing protocol, token-based congestion control and modified token-based congestion control

\section{INTRODUCTION}

Opportunistic Networks (OPPNets) are subclass of delay tolerant network where communication opportunity are intermittent and connected paths rarely exist between the source node and the destination node. The link performance in an OppNets is always subject to frequent disruption. Thus, the traditional networks, such as transmission control protocol and internet protocol (TCP/IP) will fail in this type of extreme environment because contemporaneous end-to-end path between the source and the destination node may only exist briefly and unpredictable period[1].

The OppNets lack the potential to provide contemporaneous end-to-end connectivity between the nodes and extreme latencies because of long propagation delay and intermittent connectivity demands for mechanism to control the network, which are essentially different from what is obtainable on the Internet [2]. In fact, the control function provided by the Internet's (TCP) are all accomplished at the connected path over a logical connection built between the sender and the receiver. Since OppNet has 'connectivity challenged' issues, network control in OppNets ought to be implemented on hopby-hop platform. This necessitates for custody transfer [3] and store-carry-and-forward[4] approaches that have been proposed for OppNet. This demands that OppNet nodes ought to buffer the packets in persistent storage for unpredictable period and move about until best next hop is discovered. In view of this, congestion control is considered fundamental to ensure that OppNet nodes are free from congestion and can serve as relays to aid in message delivery process. OppNets have attracted much research efforts in the area of security and routing[5]. Meanwhile, one can understand that the convergence layer for OppNets still depends heavily on TCP. Therefore, the congestion control techniques contained in the TCP cannot adapt to the dynamic evolving topology presented by the OppNets. Thus, finding an appropriate congestion control measure for OppNet is deemed a critical issue. The following sections discuss about the literature survey, comparison between congestion controls strategies. Followed by the modifying token -based congestion control and the flowchart provided. Subsequently, is the conclusion and directions for future works in the fields.

\section{LITERATURE SURVEY}

In OppNets, to transmit packets several routing techniques are proposed, and a lot of research efforts are taking place in this domain, among which are broadly classified as flooding strategies and forwarding strategies[6]. However, unlike other traditional protocols implemented in TCP, assume link is already established before forwarding the packet. In OppNets, epidemic routing protocol[7] is widely utilized due to its features of transmitting non-fragmented packets in First-InFirst-Out fashion. This has enabled the epidemic router to have an optimal delivery probability and low latency as a function of buffer size as well as bandwidth. Thus, it performs better in terms of QoS. Despite the fact that it has this distinguishing feature to transmit packets to either single or several destinations[8], it has a substantial drawback of overloading a neighboring node with redundant amount of duplicated packets and making it vulnerable to congestion. Consequently, drop a significant any incoming packets. To conquer this condition, different congestion control techniques have been proposed and the implementation of congestion control mechanism that is routing protocol independent, among which some are highlighted as follow: 


\subsection{Congestion Control Strategy for OppNet Based on Message Value[9]}

It is a reactive congestion control strategy based on Message Deleting and Transferring (MDAT), which is triggered after the occurrence of congestion within a node. It operates in a manner that the congested node computed the storage value of each message as a function of forwarding probability and

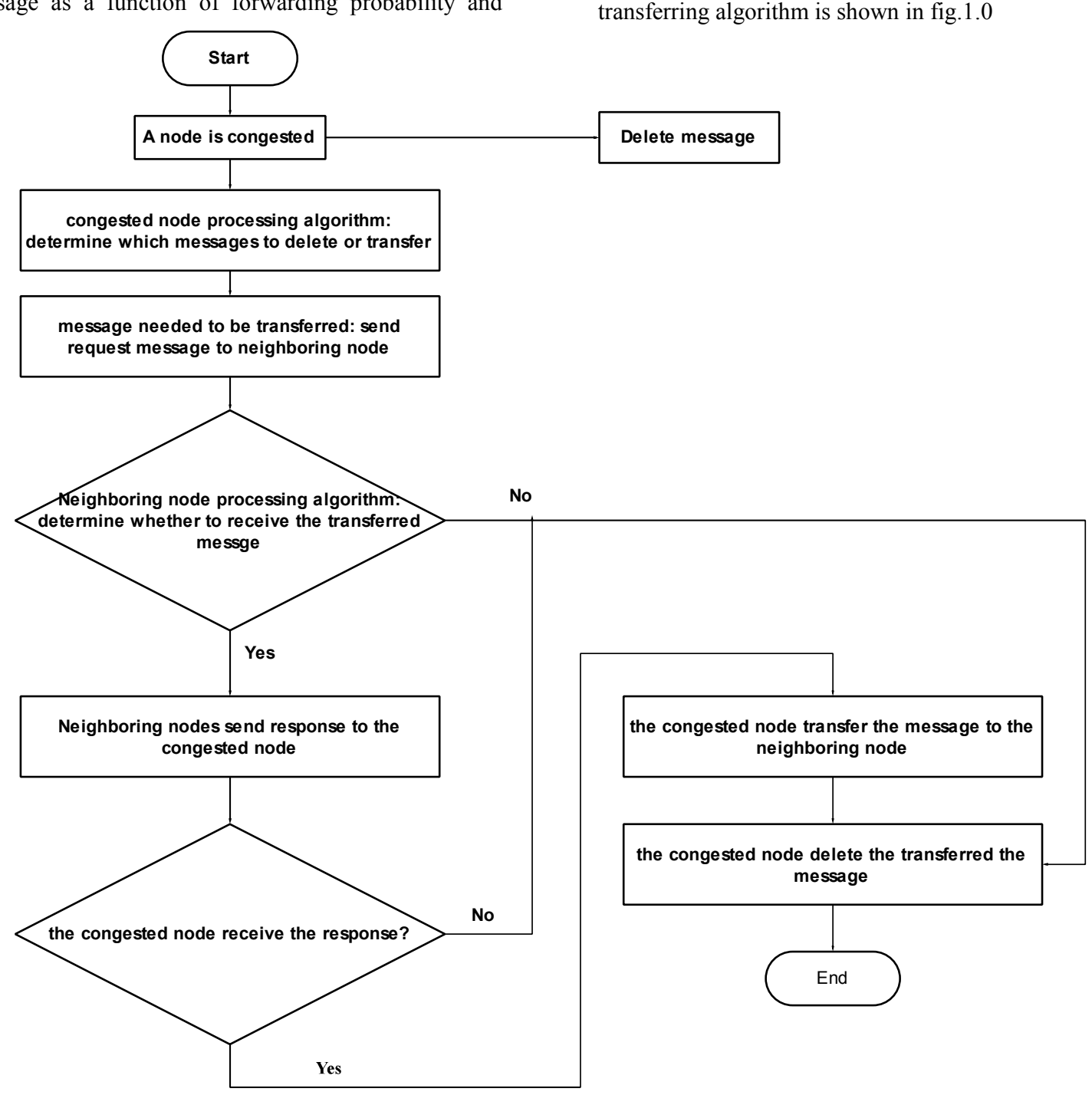

Fig 1: flow chart for MDAT algorithm[9]

A node accepts the messages if it has maximum receiving value for all incoming messages or else it checks for congestion. At this point, the Authors focused on congested node processing algorithm and neighboring node-processing algorithm. Packets are discarded if it has least storage value and minimum receiving value in the buffer of the congested node and the receiving node in order to address congestion. The flow chart of this strategy is shown in fig.1.0

\subsection{Exploiting social preference for congestion control in OppNet [10]}

In line with the message deleting and transferring, exploiting Congestion Control with Adjustable Fairness (CCAF) algorithm is also a reactive method built on any utility-based routing protocol to enhance maximum delivery rate and minimize end -to-end delay at the disbursement of a sensible decrease in fairness. The algorithm used normalized utility and normalized residual space to determine the relay node to message time-to-live. The message with the least storage value is erased. The relay node determined the receiving value of the message based on the forwarding probability and its free buffer capacity. Thus, prompting the congested node to transfer the packet with maximum receiving value. Spray and Focus routing is used for forwarding packets between contacting nodes. The flow chart for message deleting and transferring algorithm is shown in fig.1.0 


\subsection{Token based congestion control for DTNs[5]}

Different from the above congestion control strategies, TBCC is a congestion Avoidance technique, which is a proactive method. It is only the node holding a valid token can injects message into the network, which is somehow alike to Token Ring. The tokens are initially circulated evenly and then randomized. The essence of TBCC is matching the quantity of data entering the network with the network capacity and this indicates that any network or nodes should accepts as much data as it could forward. Contrast to Token Ring, TBCC only requires token to put data into the network and is recovered when the message leaves the network in favorable or unfavorable manner. The amount of tokens to be disseminated in the network differs; beside it has means of sharing the extra tokens with the neighboring nodes. The TBCC is routing algorithm independent compared with MDAT and CCAF. When simulated through discrete event simulator (DES), it showed better performance in terms dropped message and network transit time, which is directly proportional to the connectedness between the nodes via the greatest connected component (GCC) metric. It outperforms other congestion control mechanisms considered so far because it avoid congestion before it happens. Dropping message when a node's buffer is full has significant impact on the network performance and some nodes could be stressed, while others may not actively take part in message delivery process. This could results to ineffectiveness in resource utilization and unfairness in the network, which is not favorable. Figure 2, illustrates the TBCC flow chart.

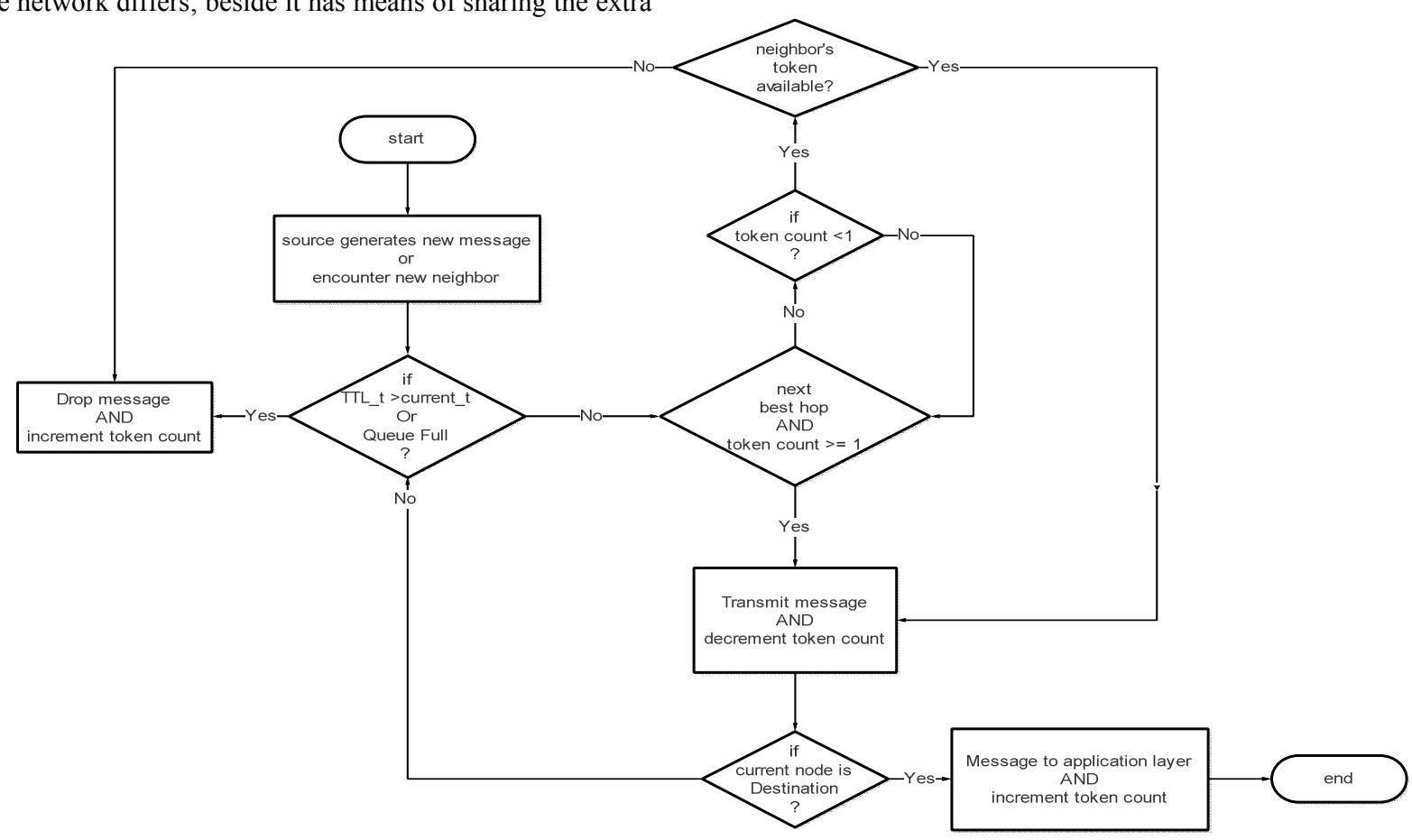

Fig.2: Flow chart for TBCC algorithm[11]

Parameters

TTL_t: Time to live timer

Current_t: Current timer

\section{COMPARISON BETWEEN THE STRATEGIES CONSIDERED}

The conversed strategies are compared, and brought together in tabular form as depicted in Table 1, which are categorized and elucidate the difference inferred between them. The categories are expounded as follows:

Table1: Comparison between the strategies considered

\begin{tabular}{|l|l|l|l|}
\hline \multirow{2}{*}{ Strategies } & \multicolumn{3}{|c|}{ categories } \\
\cline { 2 - 4 } & Type & Method & Establish on? \\
\hline MDAT & $\begin{array}{l}\text { Congestion } \\
\text { control }\end{array}$ & Reactive & $\begin{array}{l}\text { Determine the storage value of each message and delete the message with the } \\
\text { least storage value and forward the message with maximum receiving value }\end{array}$ \\
\hline CCAF & $\begin{array}{l}\text { Congestion } \\
\text { control }\end{array}$ & Reactive & $\begin{array}{l}\text { Used normalized utility and normalized residual space to define the rely node to } \\
\text { forward message }\end{array}$ \\
\hline TBCC & $\begin{array}{l}\text { Congestion } \\
\text { control }\end{array}$ & Proactive & Matching the amount of data entering the network to the total network capacity \\
\hline
\end{tabular}




\subsection{Established on strategies}

These mechanisms are triggered when the node suffers from congestion during storage management. Else, it buffers the message until opportunistic contact exist to forward the packet to the destination. These are useful in optimizing the delivery rate of packets without any issue. Due to substantial drawback of OppNet Epidemic Routing Protocol, several OppNets protocols are developed to route packets from source to destination successfully. CCAF operates on the dynamically adjusting the tunable parameters through the social preference of the nodes to provide the trade-off between efficiency and fairness, that matched the desired network performance. MDAT works on congested node processing algorithm and the receiving node-processing algorithm. Likewise, TBCC is a technique where by a node ought to have valid token in order to put message into the network or another neighboring node. In essence, it attempts to monitor the amount of traffic entering the network to the network capacity. Tokens are initially circulated and then randomized.

\subsection{Established on Type and Method}

Fundamentally, there are two kinds of congestion control techniques[11]: 1) Congestion control is a reactive method that permits the occurrence of congestion and attempts to eliminate it after detection. 2) Congestion Avoidance is a proactive method that attempts to avoid congestion prior to its occurrence. CCAF and MDAT congestion control strategies belong to the reactive method while $\mathrm{TBCC}$ is proactive method.

\section{MODIFIED TOKEN BASED CONGESTION CONTROL (mTBCC)}

Any networks loosing packets during communication is a serious problem that deserve urgent attention. Considering the issue of OppNets, where congestion leads to packet drops, is vital as establishing connection between nodes where connected routing is not guaranteed. To overcome the limitation of epidemic routing protocol, which is overloading the neighboring nodes with redundant packets. There is every need to try to avoid packet drop in the network as much as possible by utilizing more active congestion control method. To strengthen the development of modified token-based congestion control mechanism, hybrid technique is employed comprising of TBCC and Adaptive Forwarding approach to address congestion within a node.

In the event of congestion in a node and the node has to discard the incoming message(s) that can be based on any one of those congestion control strategies, will impede the network performance. In addition, network performance depends greatly on the effectiveness of resource utilization and fairness in the network. Therefore, before dropping packet, especially in one copy forwarding protocol (PHoPHET), the incoming message should be rerouted from the congested node to congestion- free node taking into account of the largest available free buffer space and migration cost. This brings the message closer to the destination in the absence of end-to-end connectivity between the source and destination node. The conclusion drawn from the comparative study is that congestion avoidance method is favored over the congestion control method as pointed out in TBCC[5], since it maintains the network in a safe state, and do way from congestion through appropriate action before it happens.

Fig.3, discusses the proposed development of the modified token-based congestion control with adaptive forwarding mechanism (mTBCC) algorithm to avoid as well as control congestion in OppNet node taking cognizance of the largest available free buffer space and migration cost. Equations 1 and 2 describes the buffer threshold and the migration threshold respectively.

$$
\begin{aligned}
& M C t h=T C+S C \\
& B S t h=L i(\max )+\mathrm{lc}
\end{aligned}
$$

Where

MCth: migration cost threhold, TC: transmission cost, SC: storage cost

Li (max): maximum message length, lc: packet overhead length

BSth: buffer size threshold

The node can be source, destination or relay forwarding node between the source and destination node. The following algorithm describe how message enters into the network and the action taken when message leaves the network. Coupled with the flow chart of Fig.3, can be utilized to avoid and control congestion in OppNet as follows:

1) Source node encounters new neighbor(s)

Source queries routing protocol to determine if one of the new neighbors is the best next hop for message held in the application queue.

if best next hop $\neq$ NULL AND my token count $\geq 1$ then next hop

decrement token count and transmit message to best

else if token count $<1$ then

query neighbors for extra token

if extra token is available then

decrement neighbors token count and transmit message to best next hop

end if

end if

2) Source node generates new message

Source queries routing protocol to determine if one of the new neighbors is the best next hop for message held in the application queue.

if best next hop $\neq$ NULL AND my token count $\geq 1$ then

decrement token count and transmit message to best next hop

else if token count $<1$ then

query neighbors for extra token

if extra token is available then

decrement neighbors token count and transmit message to best next hop

end if

3) Message dropped in the network Upon receiving message node checks

if queue State $=$ FULL then

Redirect the traffic from congested node to congestion free node. If buffer of all nodes is full, drop the message AND increment token count. 
end if

4) Message TTL timer expires

Upon routing beacon update receipt for each message in the queue check

if TTL_timer $>$ Current_timer then

Drop message AND increment token count

end if
5) Message reaches destination

Upon receiving message node checks

if message Destination $=$ this then

Pass the message to application layer AND increment token count

end if

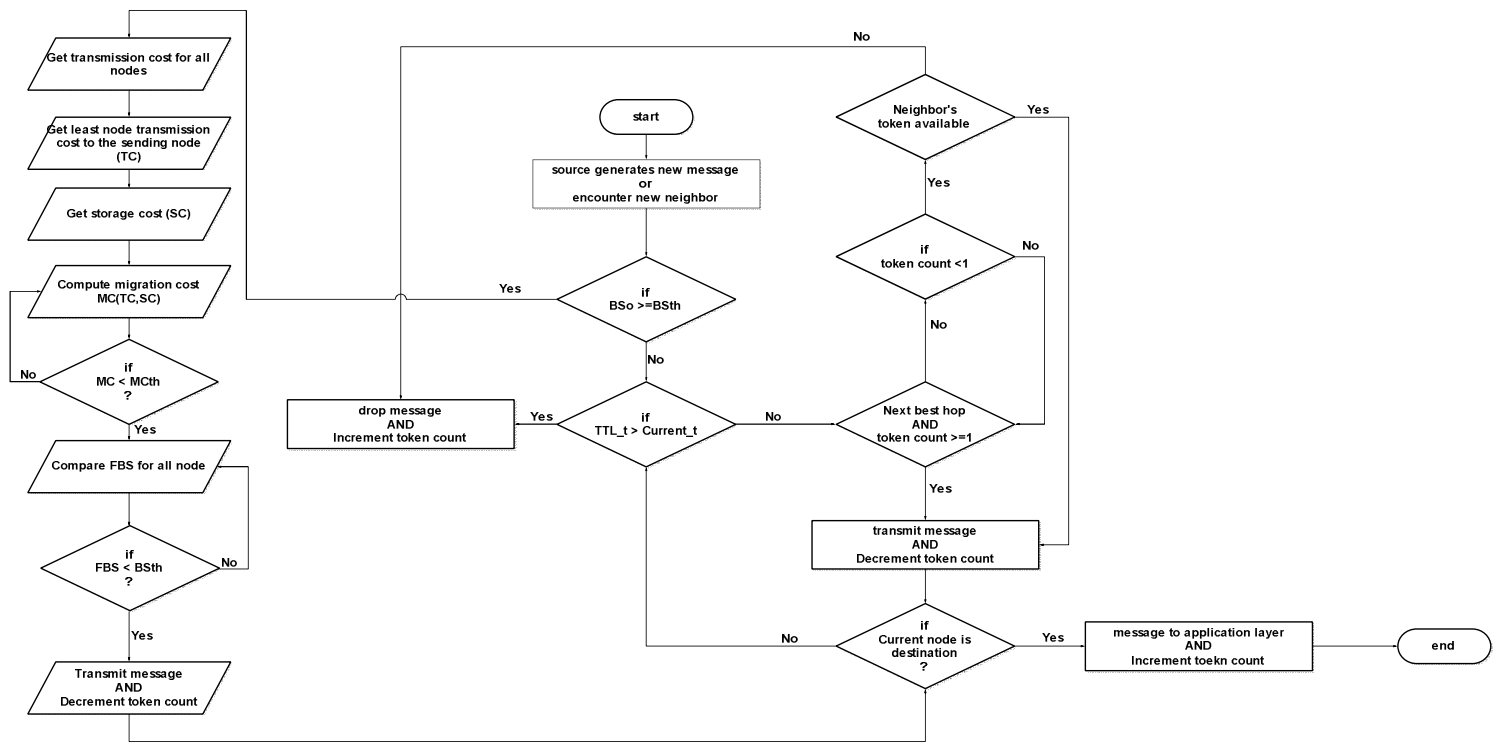

Fig.3: flow chart for mTBCC algorithm

Parameters

BSo: Buffer size over
BSth: Buffer size threshold
TTL_t: Time to live timer
Current_t: Current timer
MC: Migration cost
TC: Transmission cost
SC: Storage cost
MCth: Migration cost threshold
FBS: Free buffer space

\subsection{Environment Setup and Discussion}

The networks of interest are generated from the greatest connected component mobility model comprising of sixty mobile nodes in addition to the random waypoint mobility model operating in a Helsinki region $(4500 \times 3400 \mathrm{~m})$ with a range of $5 \mathrm{~m}$. Nodes move within this region with speed of $5 \mathrm{~m} / \mathrm{s}$. the routing protocol is based on predictability probability. Nodes update their routing table with predictability metric and compares neighboring predictability metric values for the destination with the node's value. The node with maximum value for predictability metric is considered the next best hop. The main simulation parameters are presented in table 2 .
Table 2: Simulation Parameters

\begin{tabular}{|c|c|}
\hline Parameter & Value \\
\hline Simulation area & $4500 \times 3400 \mathrm{~m}$ \\
\hline Transmission range & $5 \mathrm{~m}$ \\
\hline Transmission speed & $2 \mathrm{Mbps}$ \\
\hline Total number of nodes & 60 \\
\hline Message size & $5 \mathrm{~KB}-3 \mathrm{MB}$ \\
\hline Message creation interval & $25-35 \mathrm{~s}$ \\
\hline Simulation time & $43200 \mathrm{~s}$ \\
\hline Node movement speed & $4.5-5 \mathrm{~m} / \mathrm{s}$ \\
\hline Node buffer size & $5 \mathrm{MB}, 20 \mathrm{MB}, 60 \mathrm{MB}$, \\
& $200 \mathrm{MB}, 300 \mathrm{MB}$ \\
\hline
\end{tabular}

\section{CONCLUSION AND FUTURE WORK}

OppNets are one type of DTNs where communication opportunity occurs intermittently and contemporaneous endto-end connectivity rarely exists between the source and destination node. OppNets are vulnerable to recurrent link disruption, yet still capable to establish connections between the nodes, which is challenging and expensive. Thus, it has several application areas that is broadly researched, developed and implemented. There are different routing techniques in OppNets, amongst which is epidemic routing that is widely used due to its performance features. However, it congests communication node easily and extreme packet dropping. Meanwhile, some congestion control strategies have been 
developed using different routing protocol to overcome the limitation of the epidemic routing protocol. These are considered and compared. The mTBCC algorithm is proposed to avoid and control congestion in OppNets. In future work, we will study dynamic creation of token and deletion using localized detection. The concept of token scheme is associated to the network capacity and there has been little efforts in determining the OppNets capacity.

\section{REFERENCES}

[1] Vidya, K. and S. Hemanth, Routing and congestion control strategies in opportunistic networks: A survey. International Journal For Technical Research In Engineering. 2 (11), 2347, 2014. 4718.

[2] Silva, A.P., et al. Smart Congestion Control for Delayand Disruption Tolerant Networks. in 13th Annual IEEE International Conference on Sensing, Communication, and Networking (SECON), 2016. 2016. IEEE.

[3] Fall, K., W. Hong, and S. Madden, Custody transfer for reliable delivery in delay tolerant networks. IRB-TR-03030, July, 2003.

[4] Chuah, M.-C., et al. Store-and-Forward Performance in a DTN. in Vehicular Technology Conference, 2006. VTC 2006-Spring. IEEE 63rd. 2006. IEEE.

[5] Coe, E. and C. Raghavendra. Token based congestion control for DTNs. in IEEE Aerospace Conference, 2010. 2010. IEEE.
[6] SuvarnaPatil, G. and R. Chillerge, Delay Tolerant Networks-Survey Paper. Int. Journal of Engineering Research and Applications, 2014. 4(2).

[7] Cao, Y. and Z. Sun, Routing in delay/disruption tolerant networks: A taxonomy, survey and challenges. IEEE Communications surveys \& tutorials, 2013. 15(2): p. 654-677.

[8] Singh, C., et al., Optimal forwarding in delay-tolerant networks with multiple destinations. IEEE/ACM Transactions on Networking (TON), 2013. 21(6): p. 1812-1826.

[9] Zhang, D.-y., M. Yang, and L. Cui, Congestion control strategy for opportunistic network based on message values. Journal of Networks, 2014. 9(2): p. 1132-1138.

[10] Akestoridis, D.-G., N. Papanikos, and E. Papapetrou. Exploiting social preferences for congestion control in opportunistic networks. in 2014 IEEE 10th International Conference on Wireless and Mobile Computing, Networking and Communications (WiMob). 2014. IEEE.

[11] Patil, P. and M. Penurkar. Congestion avoidance and control in Delay Tolerant Networks. in International Conference on Pervasive Computing (ICPC), 2015. 2015. IEEE. 